\title{
Cuestionario sobre el impacto de la pandemia en la atención del estrabismo y la ambliopía en Latinoamérica
}

\section{Questionnaire about the impact of the pandemic on strabismus and amblyopia care in Latin America}

\author{
Silvia Moguel-Ancheita* \\ Mira Centro Oftalmológico, Ciudad de México, México
}

\begin{abstract}
Resumen
Objetivo: Identificar la conducta y la opinión de los médicos dedicados al estrabismo y la ambliopía sobre el impacto de la pandemia de COVID-19. La preocupación por el retraso en el tratamiento de los pacientes ya diagnosticados de estrabismo y ambliopía, así como la dificultad para el estudio integral de los casos nuevos, han sido base para la aplicación del cuestionario dirigido a los países Latinoamericanos. Método: Se estableció un cuestionario de 11 preguntas dirigidas a médicos con atención a estrabismos y ambliopías, de países latinoamericanos. Se empleó la plataforma Google Drive, con los elementos gráficos bajo estadística descriptiva, y tiempo de exposición 9-12 de octubre de 2020. Estos resultados se reportaron parcialmente durante el webinar de la Asociación Europea de Estrabismo y la Asociación Internacional de Estrabismo: Impact of COVID-19 on strabismus management around the world, el 18 de octubre de 2020. Resultados: Se obtuvieron 158 respuestas de 14 países: México 37\%, Argentina 19\%, Venezuela 14\%, Colombia 13\%, Perú 5\%, Chile 3\%, Ecuador $2 \%$, y Brasil, Paraguay, El Salvador, Bolivia, Costa Rica, República Dominicana y Guatemala 1\%. Conclusiones: El cuestionario sobre el impacto de la pandemia de COVID-19 demuestra la preocupación de los países Latinoamericanos ante la dificultad en el seguimiento de los pacientes con estrabismo y ambliopía, pero con mayor intensidad en los casos nuevos y silenciados que se pudieran estar presentando actualmente, y que las situaciones de encierro, economía y dificultad de acceso a los servicios de salud puedan estar comprometiendo de modo peligroso la vida.
\end{abstract}

Palabras clave: Estrabismo. Pandemia. Ambliopía. Nistagmo. Ceguera. Discapacidad.

\section{Abstract}

Objective: To identify the behavior and opinion of doctors dedicated to strabismus and amblyopia care regarding the impact of the COVID-19 pandemic. The concern about treatment delay in patients already diagnosed with strabismus and amblyopia, as well as the difficulty for the comprehensive study of new cases, has been the basis for the application of the questionnaire aimed at Latin American countries. Methods: A questionnaire of 11 items was prepared for physicians who attend strabismus and amblyopia cases from Latin American countries. The Google Drive platform was used, with the graphic elements under descriptive statistics and an exposure time from October 9-12, 2020. These results were partially reported during the webinar of the European Strabismological Association and International Strabismological Association: Impact of COVID-19 on strabismus management around the world, October 18, 2020. Results: We obtained 158 responses from 14 countries: Mexico

\section{Correspondencia:}

*Silvia Moguel-Ancheita

San Francisco, 1626-605

Col. Del Valle, Alc. Benito Juárez

Fecha de recepción: 28-10-2020

Fecha de aceptación: 08-01-2021

C.P. 03100, Ciudad de México, México

E-mail: smoguel@ prodigy.net.mx

0187-4519/@ 2021 Sociedad Mexicana de Oftalmología. Publicado por Permanyer. Este es un artículo open access bajo la licencia CC BY-NC-ND (http://creativecommons.org/licenses/by-nc-nd/4.0/).
Disponible en internet: 05-05-2021 Rev Mex Oftalmol. 2021;95(3):107-117

www.rmo.com.mx 
$37 \%$, Argentina 19\%, Venezuela 14\%, Colombia 13\%, Peru 5\%, Chile 3\%, Ecuador 2\%, and Brazil, Paraguay, El Salvador, Bolivia, Costa Rica, Republic Dominican and Guatemala 1\%. Conclusions: The questionnaire on the impact of the COVID-19 pandemic demonstrates the concern of Latin American countries regarding the difficulty in monitoring patients with strabismus and amblyopia; but with greater severity in the new and silenced cases happening right now, and that the situations around confinement, economy, and difficulty of access to health services, can be compromising health in a dangerous way.

Key words: Strabismus. Pandemic. Amblyopia. Nystagmus. Blindness. Impairment.

\section{Introducción}

En diciembre de 2019 inició en China la enfermedad provocada por el coronavirus del síndrome respiratorio agudo grave tipo 2 (SARS-CoV2), denominada COVID-19 y declarada pandemia el día 11 de marzo del año 2020 por la Organización Mundial de la Salud'.

Latinoamérica fue afectada con posterioridad a los países asiáticos y europeos. Para el 9 de octubre de 2020, fueron 9.876.651 los casos de COVID-19 registrados en América Latina y el Caribe, considerándose a Brasil como el país más afectado, con más de 5 millones de casos confirmados. Colombia se ubicó en segundo lugar, con alrededor de 886.000 infectados, y México, por su parte, registró un total de 804.488 casos, seguidos de Argentina, Perú, Chile y Ecuador ${ }^{2}$.

El día 18 de marzo, la American Academy of Ophthalmology sugirió a los oftalmólogos el cese inmediato de las actividades no urgentes basándose en las recomendaciones del American College of Surgeons, así como medidas higiénicas estrictas y un adecuado control para las consultas presenciales, además de haber promovido el uso de la consulta por teleconferencia. Dentro de las recomendaciones se han mencionado el manejo de un cuestionario de antecedentes de riesgo y exposición, el espaciamiento de personas en la sala de espera, las consultas de corto tiempo, medidas de precaución en la exploración con la lámpara de hendidura, evitar la comunicación hablada, el control de cubrebocas de calidad quirúrgica, la limpieza de las áreas posterior a cada paciente y, en caso de requerirse gotas para la exploración, que sean unidosis ${ }^{3}$.

La recopilación de las recomendaciones para el manejo de pacientes que requieren atención oftalmológica durante la pandemia por SARS-CoV-2 emitida por la Sociedad Mexicana de Oftalmología incluye, incluso, evaluar la necesidad de realizar tonometrías o posponerlas a criterio del médico; en caso de personas que no tengan urgencias oftalmológicas, que sean atendidos por vía telefónica o aplicaciones digitales, además de recomendarles a esperar que pase la crisis sanitaria y comunicarse en caso de que su situación visual cambie; y respecto a las cirugías oftalmológicas, quedaron asignadas como electivas aquellas que, de no realizarse, no lleven riesgo de pérdida visual a corto plazo, rubro en el que quedó cotejada toda cirugía de estrabismo 4 .

Con esta información y con los cambios que se han presentado en los siguientes 8 meses de crisis sanitaria en los diferentes países de Latinoamérica y el Caribe, se realizó una encuesta con el objetivo de identificar más adecuadamente la conducta y la opinión de los médicos dedicados a estrabismo y ambliopía, considerando que se puede percibir una preocupación generalizada, toda vez que ambos padecimientos pueden formar parte de síndromes complejos en la salud integral de los pacientes, y que incluso por sí solos generan una alteración visucerebral mayor tanto por el retraso en su diagnóstico como por la suspensión de su tratamiento.

\section{Método}

Se estableció un cuestionario de 11 preguntas dirigidas al impacto que puede haber provocado la pandemia en el manejo de los estrabismos y las ambliopías en los diferentes países de Latinoamérica. El cuestionario se envió a los representantes en los países de Latinoamérica participantes en los Trabajos de Discapacidad Visual que se realizan anualmente a través del Centro Mexicano de Estrabismo. Las preguntas fueron redactadas específicamente para la identificación de los problemas en los diagnósticos y los tratamientos de los estrabismos en sus diversos tipos, así como en la ambliopía como causa de discapacidad visual. Se ofreció la posibilidad de resguardar los nombres y la información de los encuestados, ante la posibilidad de que pudieran agregar comentarios al margen. La aplicación del cuestionario fue a traves de Google Drive y se emplearon los elementos gráficos del mismo bajo estadística descriptiva, con un tiempo de exposición del 9 al 12 de octubre de 2020. Estos resultados se reportaron parcialmente durante el webinar de la Asociación Europea de Estrabismo en conjunto con la 
1) Ante el cierre de servicios de salud, su consulta privada se ha afectado del siguiente modo:

a) Ha aumentado

b) Se redujo

c) Sigo sin dar consulta

3) La cirugía de estrabismo para usted:

a) No ha suspendido por cuarentena

b) Solo opera urgencias

c) Sigue sin programar cirugía

5) Respecto a sus pacientes con tratamiento por ambliopía:

a) Los ha citado regularmente

b) Le han contactado por redes, WhatsApp, celular

c) No los pudo ver, hasta estar citados en su consulta programada

7) En su exploración actual oftalmológica:

a) La realiza igual que siempre

b) Usa gotas ciclopléjicas solo en caso necesario, tonometría solo en caso necesario

c) Ha diferido toda exploración que requiera contacto directo, gotas, tonometría

9) Respecto a la posibilidad de revisión a distancia por telefonía, videoconferencia, etc.:

a) No la usa, su consulta sigue siendo presencial

b) Combina consulta virtual con presencial

c) Solo virtual, no realiza consulta presencial

11) ¿Cuánto ha afectado la preparación de los residentes en la oftalmología?

a) Lo mínimo, se han mantenido actividades oftalmológicas todo el año

b) $50 \%$

c) Severo, se planea reponer próximamente
2) ¿Consideraría incluir de modo permanente en su atención las video consultas?

a) No, prefiero siempre presencial

b) Solo en casos especiales

c) Definitivamente sí

4) ¿Por la pandemia los casos de diagnóstico grave se han afectado?

a) No, se han diferido y estudiado adecuadamente

b) Se ha retrasado únicamente el control de pacientes estables

c) Se ha afectado gravemente la detección de estrabismos por causas graves como tumores, daño cerebral, etc.

6) En su consulta actual, ¿qué impacto ha notado en la evolución de sus pacientes con ambliopía y estrabismo?

a) Sin afectación, los he seguido cercanamente

b) Poco impacto, aunque no los haya visto cercanamente

c) Gran impacto, la evolución se ha visto agravada

8) ¿La conversión a COVID de los hospitales ha afectado la atención de los estrabismos y ambliopías?

a) Continúan sin cambios, se atienden normal

b) Se dirigieron a otras unidades de atención no COVID

c) Se suspendió la consulta programada hasta nuevas indicaciones

10) Respecto a los estudios de sus pacientes para integrar un diagnóstico:

a) Sin problemas, continúo solicitando OCT, RMN, TAC, lo que sea necesario

b) Solicito solamente lo verdaderamente urgente, evito estudios

c) Muy afectados, no hay citas o los pacientes no pueden por la economía

Figura 1. Cuestionario para conocer el impacto de la pandemia en la atención de los estrabismos y las ambliopías en Latinoamérica.

Asociación Internacional de Estrabismo, durante el primer encuentro de otoño: Impact of COVID-19 on strabismus management around the world, llevado a cabo el 18 de octubre de 2020 (Fig. 1).

\section{Resultados}

Se obtuvieron 158 respuestas de médicos que participan en los tratamientos de estrabismo y ambliopía de su localidad, de 14 países Latinoamericanos, con las siguientes participaciones: México 37\%, Argentina $19 \%$, Venezuela $14 \%$, Colombia $13 \%$, Perú $5 \%$, Chile $3 \%$, Ecuador $2 \%$, y Brasil, Paraguay, El Salvador, Bolivia, Costa Rica, República Dominicana y Guatemala 1\% (Fig. 2).
Respecto a la pregunta 1 (157 respuestas), el 83,4\% consideró que la consulta privada se ha reducido, el $13,4 \%$ refiere que ha aumentado y el $3.2 \%$ permanece con la consulta cerrada hasta el 12 de octubre (Fig. 3).

Acerca de la pregunta 2 (158 respuestas), el 63.9\% considera que la atención telefónica la usaría solamente en casos especiales, el $29.1 \%$ prefiere la consulta presencial y solo el $7 \%$ planea quedarse con consultas telefónicas o videoconferencias una vez pasada la crisis sanitaria (Fig. 4).

Respecto a la pregunta 3 (158 respuestas), el 33.5\% no ha suspendido la cirugía de estrabismo por cuarentena, el 39.2\% únicamente realiza cirugía en urgencias y el $27.2 \%$ tiene canceladas las cirugías de estrabismo (Fig. 5). 


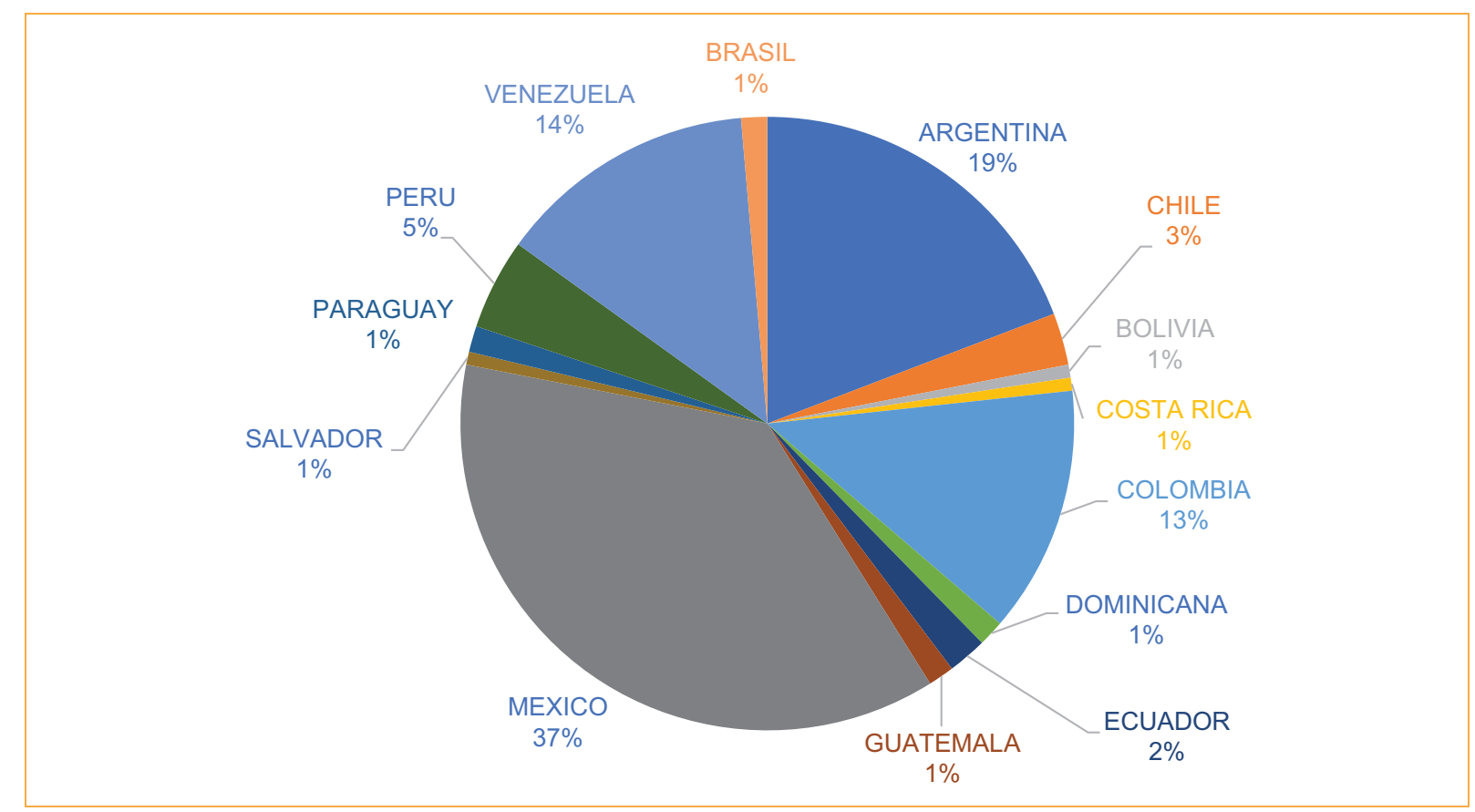

Figura 2. Participación de países latinoamericanos en el cuestionario del impacto de la pandemia en la atención de los estrabismos y las ambliopías.

\section{Ante el cierre de servicios de salud, su consulta privada se ha afectado del siguiente modo} 157 respuestas

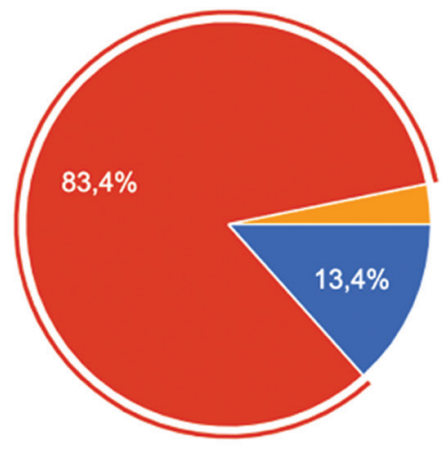

Ha aumentado

Se redujo

Sigo sin dar consulta

Figura 3. Afectación de la consulta ante la pandemia.

En la pregunta 4 (157 respuestas), el 33.1\% considera que los casos de estrabismo relacionados con eventos graves se han podido estudiar adecuadamente, el $49 \%$ considera que el retraso ha afectado a cuadros estables y el $17.8 \%$ considera que se ha afectado severamente la detección de estrabismos asociados a causas graves (Fig. 6).
En la pregunta 5 (158 respuestas), el $46.8 \%$ no ha podido saber de sus pacientes con ambliopía, el 33.5\% los ha podido vigilar por vías virtuales y el $19.6 \%$ los ha vigilado con citas normales (Fig. 7).

En relación con la pregunta 6 (156 respuestas), el $54.5 \%$ considera que el impacto en la evolución de la ambliopía en sus pacientes durante la pandemia 


\section{Consideraria incluir de modo permanente en su atención las video consultas?}

158 respuestas

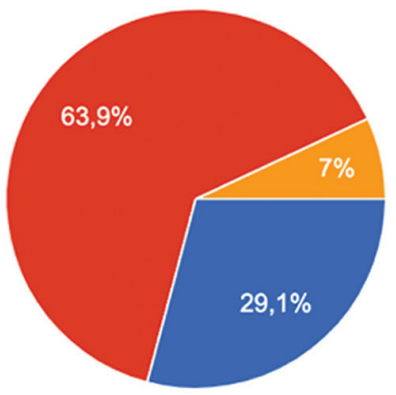

No, prefiero presencial

Solo en casos especiales

Me quedaré con videoconsultas pasada la pandemia

Figura 4. Permanencia de las video consultas.

\section{La cirugia de estrabismo para usted}

158 respuestas

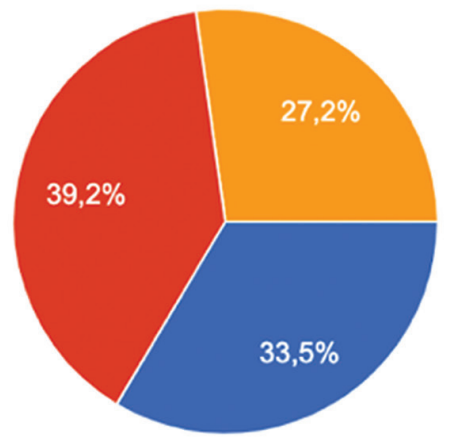

No se ha suspendido por cuarentena

Sólo opera urgencias

Sigue sin programar cirugías

Figura 5. Afectación en la cirugía de estrabismo.

\section{Por la pandemia los casos de diagnóstico grave se han afectado?}

157 respuestas

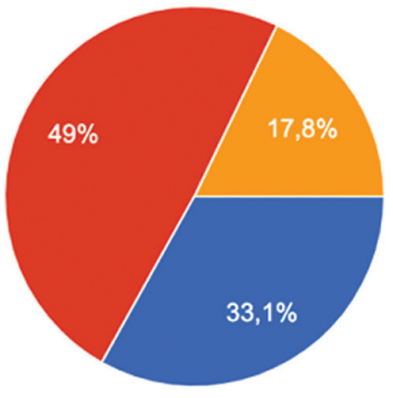

No, se han diferido y estudiado adecuadamente

Se ha retrasado únicamente el control de pacientes estables

Se ha afectado gravemente la detección de estrabismos por causas graves, como tumores, daño cerebral, etc

Figura 6. Efecto de la pandemia en los casos de diagnóstico grave. 


\section{5) Respecto a sus pacientes con tratamiento por ambliopía}

158 respuestas

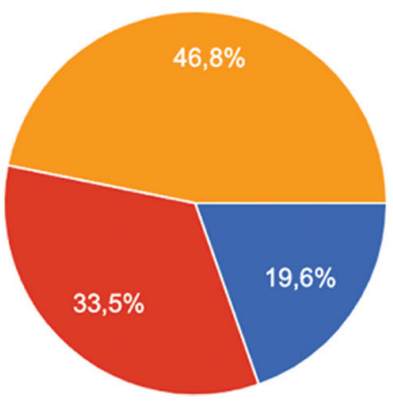

Los ha citado regularmente

Le han contactado por redes,

WhatsApp, celular

No los pudo ver, hasta estar citados en su consulta programada

Figura 7. Tipo de vigilancia realizada de pacientes con ambliopía.

6) ¿En su consulta actual qué impacto ha notado en la evolución de sus pacientes con ambliopia y estrabismo?

156 respuestas

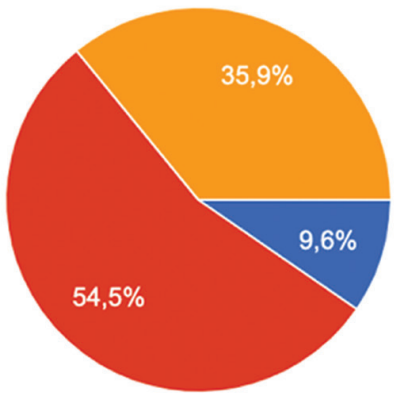

Sin afectación.

Poco impacto. Los he seguido cercanamente

Gran impacto, la evolución se ha visto agravada.

Figura 8. Efecto de la pandemia en la ambliopía ante la falta de consultas de seguimiento.

\section{7) En su exploración actual oftalmológica actual? .}

158 respuestas

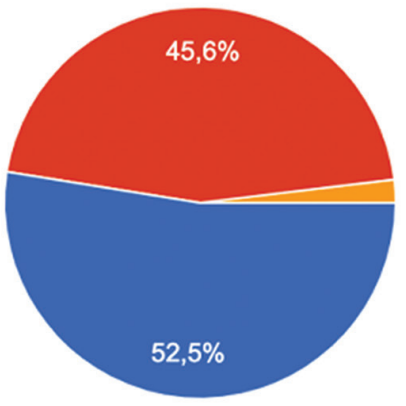

La realiza igual que siempre

Usa gotas ciclopléjicas sólo en caso necesario, tonometría sólo en caso necesario

Ha diferido toda exploración que requiera contacto directo, gotas, tonometría

Figura 9. Cambios en la exploración actual al evitar el contacto directo o el uso de gotas 
8) ¿La conversión a Covid de los hospitales ha afectado la atención de los estrabismos y ambliopias?

153 respuestas

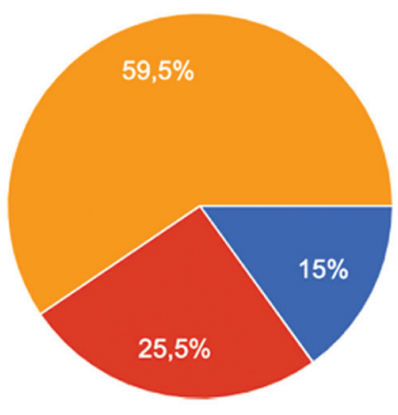

Continúan sin cambios, se atienden normal

Se dirigieron a otras unidades de atención No Covid

Se suspendió la consulta programada hasta nuevas indicaciones

Figura 10. Afectación de la consulta por la conversión hospitalaria a COVID-19.

9) Respecto a la posibilidad de revisión a distancia por telefonia, videoconferencia, etc.

158 respuestas

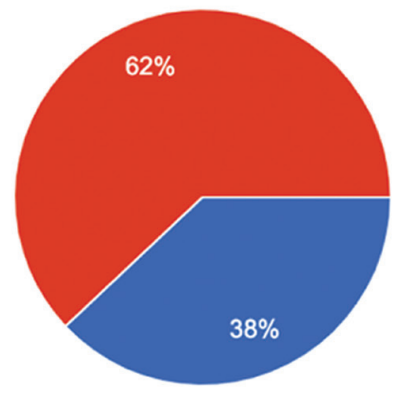

No la usa, su consulta sigue siendo presencial

Combina consulta virtual con presencial

Solo virtual, no realiza consulta presencial

Figura 11. Uso actual en pandemia de las teleconsultas.

10) Respecto a los estudios de sus pacientes para integrar un diagnóstico:

158 respuestas

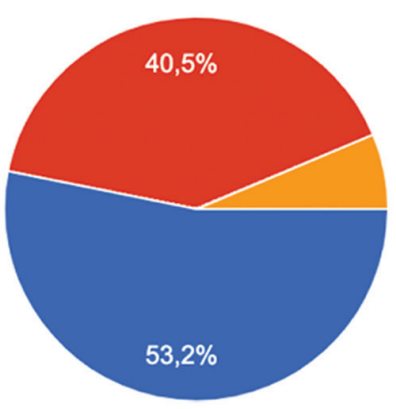

Sin problemas, continúo solicitando OCT, RMN, TAC, lo que sea necesario

Solicito solamente lo verdaderamente urgente, evito estudios

Muy afectados, no hay citas o lo pacientes no pueden por la economía

Figura 12. Afectación en la capacidad de estudios de gabinete y laboratorios para sus pacientes. 


\section{1) ¿Cuánto ha afectado la preparación de los Residentes en la Oftalmologia?}

140 respuestas

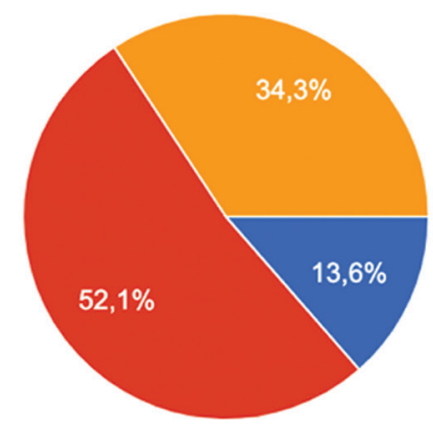

Lo mínimo. Se han mantenido actividades oftalmológicas todo el año

$50 \%$

Severo, se planea reponer próximamente

Figura 13. Afectación de la pandemia en las sedes formadoras de residentes en oftalmología.

resulta bajo, el $35.9 \%$ refiere que ha ocurrido un gran impacto generando un agravamiento en la ambliopía, y el $9.6 \%$ considera que no ha afectado de ningún modo (Fig. 8).

En la pregunta 7 (158 respuestas), el $52.5 \%$ refiere que realiza la exploración de sus pacientes de modo normal sin evitar especialmente el uso de gotas o de contacto directo, el $45.6 \%$ menciona que usa gotas si es altamente requerido y el $1.9 \%$ ha diferido todo uso de gotas o de exploración de contacto directo (Fig. 9).

En relación con la pregunta 8 (153 respuestas, considerando no respondedores a los médicos que no tienen relación con atención hospitalaria), el 59.5\% menciona que por la conversión de los hospitales a COVID-19 se suspendió toda la consulta programada, el $25.5 \%$ que la conversión no afectó a los tratamientos de estrabismo o ambliopía porque fueron a otras unidades de salud no COVID-19, y el 15\% continuó la consulta de modo normal (Fig. 10).

En la pregunta 9 (158 respuestas), el $62 \%$ de los médicos ha usado atención médica combinada telefónica y presencial, el $38 \%$ no usa atención virtual (solo presencial) y ninguno ha atendido únicamente de modo virtual (Fig. 11).

En relación con la pregunta 10 (158 respuestas), el $53.2 \%$ continúa solicitando sin notar dificultades estudios de gabinete o exámenes a sus pacientes, el $40.5 \%$ refiere que evita solicitar estudios a menos que sea muy necesario, y el $6.3 \%$ responde que ha sido difícil estudiar a sus pacientes por falta de espacios o por daño a la economía (Fig. 12).
La pregunta 11 fue agregada ante las quejas múltiples de los compañeros médicos que se encuentran realizando la alta especialidad oftalmológica en los hospitales (140 respuestas, considerando no respondedores a los médicos que no tienen relación con sedes formadoras de especialidad), y el $52.1 \%$ refiere un daño del $50 \%$, el $34.3 \%$ refiere una importante afectación y que se planea recuperar posteriormente, y el $13.6 \%$ menciona que la afectación ha sido mínima porque continúan con procedimientos propios de su programa académico (Fig. 13).

\section{Discusión}

El presente cuestionario permite notar la clara disminución en la atención de los pacientes con estrabismo y ambliopía, tanto en la consulta como en la cirugía, en especial cuando esta ha sido determinada en diferentes guías clínicas como «electiva». Prácticamente la mitad de los médicos no ha podido realizar un seguimiento de sus pacientes que se encuentran en tratamientos de ambliopía y estrabismos, y una tercera parte de los encuestados considera que el impacto de la pandemia en la falta de atención de estos pacientes ha resultado importante.

Las recomendaciones referidas sobre disminuir en la medida de lo posible la exploración cercana ha provocado la falta de exploración midriática y la tonometría, considerando que este riesgo puede ser pospuesto sin generar un daño por el retraso. Es de gran relevancia el temor en la percepción de que casos de estrabismos o nistagmos, o diferentes causas de daño ocular grave, 
como tumores, enfermedades autoinmunitarias, inflamatorias graves, metabólicas, etc., se queden guardados en casa por diversos motivos, por ejemplo, el cierre de los servicios de salud, la conversión de los hospitales, el difícil acceso a estudios de gabinete, la falta de economia en la población general e incluso, y muy importante, el temor a salir a una atención médica considerando que los síntomas puedan ser de importancia menor, ante el desconocimiento sobre los peligros en la salud que pueden estar relacionados con estrabismos nuevos o con recaídas, paralíticos, nistagmos, etc.

El médico, en gran medida, ha intentado realizar nuevas estrategias de comunicación virtual para la atención de sus pacientes, aunque podemos ver que una tercera parte de ellos no usa estos medios, y la mayoría de los que los han implementado consideran que prefieren volver a una consulta normal presencial pasada la crisis sanitaria y no se plantean dejar una fracción de su consulta bajo estos medios virtuales.

Si bien las redes sociales, los servicios de telefonía y las videoconferencias podrían ser aprovechadas en ciertas circunstancias, una exploración oftalmológica completa requiere forzosamente, hasta el momento, de la atención presencial. En el inicio de la pandemia parecía una opción viable poder usar los medios virtuales, pero el tiempo ha demostrado sus dificultades. Tomando en cuenta que la duración de la crisis sanitaria ha sido más prolongada que lo analizado por los diversos estrategas en salud, lo que parecía una oportunidad aceptable y transitoria ha cambiado por la decisión y la necesidad de la apertura de los servicios de salud para poder continuar con la atención de pacientes prioritarios.

El cierre completo de los servicios médicos ordenado por los coordinadores de salud fue una de las condiciones que impulsó las atenciones virtuales, pero la ausencia de las regulaciones legales en diversos países para este tipo de consulta virtual, no presencial, dificulta su continuidad.

Aunque la telemedicina ha sido un medio usado exitosamente en hospitales de concentración, cuenta con la característica de que en ambos lados normalmente se encuentra un profesionista de la salud realizando la presentación de los pacientes que ya han sido estudiados y que por este medio pueden ser consultados para su referencia, control o vigilancia, facilitando así sus cuidados y muy frecuentemente evitando la movilización de los pacientes y la saturación de los servicios de salud centralizados. Este tipo de actuación, así como los documentos electrónicos requeridos para el caso, han sido adecuadamente legislados, y hasta el momento las autoridades han determinado que la telemedicina es una actividad integrada a la práctica clínica, bajo estas condiciones.

Sin embargo, en muchos países, incluido México, aún no se contempla una normatividad para consultas privadas en medios virtuales, por lo que los profesionales de la salud deben estar sujetos a los principios y métodos de la práctica médica tradicional. Esto ha motivado la revisión de normas en diferentes países para promover los cambios para las denominadas teleconsultas o teleconsulta interactiva, en las que el contacto pueda ser de paciente a médico ${ }^{5-10}$.

Sin embargo, esta consulta virtual, en modo de llamadas telefónicas, teleconferencias, notas celulares, etc., que provienen del paciente, puede tener ciertas desventajas; por ejemplo, puede no contar con las condiciones completas de información del profesional, las posibilidades de exploración son bastante reducidas en el campo de la oftalmología, no se encuentra contemplada esta condición en los documentos de identidad obligatorios por las leyes sanitarias, como en México, en que el paciente debe autorizar con su firma la revisión física, además de que debe completar el consentimiento informado previo a la atención; y también deben considerarse, entre otros elementos, el riesgo de emitir una prescripción medicamentosa, el uso de imágenes o incluso cobrar por este tipo de consulta, y todo ello puede incurrir en una mala práctica médica ${ }^{11-13}$.

Se ha sugerido, en diferentes trabajos de consulta a distancia, informar al paciente de que cualquier recomendación que se le pueda dar por este medio debe considerarse provisional, que se basa en lo que el paciente está informando y que jamás podrá ser interpretada como una consulta médica ni como un diagnóstico.

Respecto a la conferencia en video, y en apoyo a la historia clínica, debe recordarse que el uso de las fotografías de un paciente está prohibido por diversas leyes y en todo caso debe existir una autorización expresa, por escrito, en la cual el paciente permita que el médico ocupe sus fotografías, bajo el proceso durante el cual el paciente tiene el derecho de elegir o descartar las que pueden ser publicadas y expresar el límite de tiempo o modo de uso de cada imagen. Así mismo, el médico también deberá hacer firmar al paciente un descargo para el uso de la imagen y una renuncia sobre los derechos económicos que involucra el uso de imágenes de terceros. Se advierte que nunca se podrá publicar una imagen de una persona menor de edad, ni se podrá legalmente hacer uso de 
imágenes de personas que no han suscrito con él el consentimiento de uso de la imagen o la autorización del apoderado legal de pacientes con incapacidad. Igualmente, respecto a la difusión de la información que ha sido favorecida por las redes sociales, los médicos deben observar la ley y las reglas que hay que cumplir para realizar una promoción, y no incurrir en retención dolosa de información ni en publicidad engañosa o falsa, ya que esto puede comprometer su responsabilidad civil y penal.

También se ha insistido sobre no emplear videoconferencias en condiciones especiales que incluyen el que se requiera una exploración física para establecer un diagnóstico, si el paciente no tiene salud mental suficiente para dar una información certera, o si tiene dificultades para la comprensión de la tecnología o de la información que pudiera otorgarle el médico ${ }^{14}$.

Existen también las advertencias internacionales en los países donde ya se ha autorizado el uso de la consulta virtual y que no exista la posibilidad de afectar la privacidad de la información si no se puede llevar control sobre terceros que escuchen la llamada, así como los riesgos de que esta sea grabada, ya que no es autorizado legalmente. Se ha hecho la sugerencia de que el médico solo debe utilizar la telemedicina en países que cuenten con jurisdicciones con licencia para emplearla, y las consultas de jurisdicciones cruzadas solo deben permitirse entre dos médicos, e incluso asegurarse de que su seguro médico incluye las normatividades que está ocupando para su teleconsulta ${ }^{15,16}$.

En México, la Comisión Nacional de Arbitraje Médico considera, entre otros factores que inciden en los errores médicos, el riesgo de una mala comunicación entre médico y paciente, un mal interrogatorio y una deficiente exploración física, así como la falta de seguimiento, una inadecuada historia clínica, inadecuados métodos de trabajo, falta de estandarización de los procesos de atención e incumplimiento de normas y de procesos de calidad ${ }^{17}$.

Otro aspecto de gran importancia durante el confinamiento obligado por la pandemia ha sido su impacto sobre padecimientos en los que se puede correr un alto riesgo de salud, y cuyo retraso en el diagnóstico puede aludirse tanto al cierre de los servicios médicos como al temor a salir de casa, lo que hemos referido como "drama silencioso». Podemos registrar, por ejemplo, la suspensión de estudios de control para la detección de cáncer de mama, el impacto en el daño cardiaco mantenido en casa (que ha alcanzado en algunas regiones de Italia hasta un 200\%), la disminución de los estudios y de los tratamientos cardiovasculares de un 40-81\% en España, etc. Estos eventos graves requerirían un aviso más allá del confinamiento sobre la advertencia de "quédate en casa", para dar a conocer los riesgos en enfermedades prioritarias de riesgo a la salud paralelas a la pandemia $^{18,19}$.

El Consejo Nacional de Población (Conapo) en México ha advertido de que el encierro provocado por la actual pandemia de COVID-19 ha generado un aumento de las necesidades insatisfechas de anticoncepción de hasta un $20 \%$, por lo que se espera un número adicional de 145,719 embarazos no deseados o no planeados en mujeres de 15 a 49 años, y de 21,575 embarazos no deseados o no planeados en adolescentes de 15 a 19 años. El embarazo adolescente conlleva una relación directa de mayor prematuridad a menor edad de la madre, con más alto índice de malformaciones y secuelas asociadas a bajo peso, con graves daños neurológicos; estos niños con nacimiento prematuro se encuentran envueltos en una época con extremadamente limitadas sedes de rehabilitación y diagnóstico, y probablemente veremos incrementarse el número de discapacitados visuales, físicos, ciegos, etc., en el siguiente año $20-22$.

Además de la frecuencia de daño visual, estrabismo, nistagmo, discapacidad visucerebral, etc., en los niños prematuros, que requieren una atención inmediata con revisión presencial que se ha visto muy limitada durante todos los meses de confinamiento, también se encuentran las personas con enfermedades metabólicas, autoinmunitarias, endocrinológicas, etc., que pueden estar sufriendo el poco acceso a sus controles, medicamentos, rehabilitación y control, y diagnóstico, aunado al aumento de la comorbilidad en condiciones de encierro, como la obesidad, el abandono de los pacientes discapacitados e incluso la ausencia de cuidadores y familiares ${ }^{23}$.

Comprendemos las limitaciones del presente análisis, tomando en cuenta las diferentes opiniones que parten de las condiciones que se viven en cada país y la falta de participación de algunos representantes de otros países latinoamericanos cuya ausencia de representación en el estudio y análisis de los estrabismos dejan vacía la posibilidad de aportar información del estado en que se encuentran, y comprendemos la imprecisión que, a pesar de su gran valor, pueda aludirse a las opiniones personales. La persistencia en la evolución de las cifras y los porcentajes observados durante el desarrollo del cuestionario permiten 
reconocer las actuales preocupaciones, por lo que consideramos que esta información adquiere valor al ser una situación palpable y evidente en diversas áreas de la salud, lo que permite extrapolar la importancia en el diagnóstico de estrabismos, especialmente los relacionados con enfermedades neurológicas, metabólicas, autoinmunitarias, etc., en las que pueda estar corriendo riesgo la vida. Sugerimos considerar las opiniones en el contexto de la población que se esté analizando, así como las concurrencias y similitudes.

\section{Conclusiones}

El cuestionario sobre el impacto de la pandemia de COVID-19 demuestra la preocupación de los países latinoamericanos ante la dificultad en el seguimiento de los pacientes que han cursado con estrabismo y ambliopía, como el retraso en su evolución; pero con mayor impacto en los casos nuevos y silenciados que se pudieran estar presentando actualmente, y que las situaciones de encierro, economía y dificultad de acceso a los servicios de salud puedan estar comprometiendo, más allá de la función visual, y de modo peligroso, la vida.

\section{Agradecimientos}

Agradezco a todos los participantes de los países latinoamericanos la colaboración para la respuesta al cuestionario; como fue asignado, no se registraron los nombres para conservar la privacidad.

\section{Conflicto de intereses}

La autora declara no tener ningún conflicto de intereses.

\section{Responsabilidades éticas}

Protección de personas y animales. Los autores declaran que para esta investigación no se han realizado experimentos en seres humanos ni en animales.

Confidencialidad de los datos. Los autores declaran que en este artículo no aparecen datos de pacientes.

Derecho a la privacidad y consentimiento informado. Los autores declaran que en este artículo no aparecen datos de pacientes.

\section{Bibliografía}

1. Gobierno de México. (Consultado el 24/10/2020.) Disponible en: https:// coronavirus.gob.mx/covid-19/.

2. STATISTA. Estado de Salud. (Consultado el 24/10/2020.) Disponible en: https://es.statista.com/estadisticas/1105121/numero-casos-covid-19-america-latina-caribe-pais/.

3. Important coronavirus updates for ophthalmologists. AAO. (Consultado el 24/10/2020.) Disponible en: https://www.aao.org/headline/alert-important-coronavirus-context.

4. Recopilación de las recomendaciones para el manejo de pacientes que requieren atención oftalmológica durante la pandemia de SARS-CoV-2. (Consultado el 25/10/2020.) Disponible en: https://smo.org.mx/archivos/ documentos/2020/Recomendaciones\%20COVID-19\%20SMO.pdf.

5. Ministerio de Salud y Protección Social. Colombia. Octubre 2019. (Consultado el 25/10/2020.) Disponible en: https://www.minsalud.gov.co/Normatividad Nuevo/Resoluci\%C3\%B3n\%20No.\%202654\%20del\%202019.pdf.

6. Programa Nacional de Telesalud. Gobierno de Chile. 2015. (Consultado el 25/10/2020.) Disponible en: https://www.minsal.cl/wp-content/ uploads/2015/08/TELESALUD webV2.pdf.

7. Ministerio de Salud y Protección Social. Colombia 2019. (Consultado el 25/10/2020.) Disponible en: https://www.minsalud.gov.co/Normatividad Nuevo/Resoluci\%C3\%B3n\%20No.\%202654\%20del\%202019.pdf.

8. Proyecto de Ley. Regulación de la Telemedicina en Argentina. Diputados 2020. (Consultado el 25/10/2020.) Disponible en: https://www4.hcdn.gob.ar/ dependencias/dsecretaria/Periodo2020/PDF2020/TP2020/3142-D-2020.pdf.

9. Norma Oficial Mexicana. NOM-024-SSA2-2010, que establece los objetivos funcionales y funcionalidades que deberán observar los productos de Sistemas de Expediente Clínico Electrónico para garantizar la interoperabilidad, procesamiento, interpretación, confidencialidad, seguridad y uso de estándares y catálogos de la información de los registros electrónicos en la salud. (Consultado el 25/10/2020.) Disponible en: http:// www.dof.gob.mx/normasOficiales/4151/salud/salud.htm.

10. Cámara de Diputados. Proposición para Norma Oficial Mexicana en materia de telemedicina. 21 de abril de 2020. (Consultado el 25/10/2020.) Disponible en: http://sil.gobernacion.gob.mx/Archivos/Documentos/2020/04/asun_4034093_20 200421 1587533515.pdf.

11. Reglamento de la Ley General de Salud en materia de prestación de servicios de atencón médica. Última reforma 17/07/2018. (Consultado el 25/10/2020.) Disponible en: http://www.diputados.gob.mx/LeyesBiblio/ regley/Reg_LGS_MPSAM_170718.

12. Norma Oficial Mexicana. NOM-004-SSA3-2012, del expediente clínico. (Consultado el 25/10/2020.) Disponible en: http://dof.gob.mx/nota_detalle_popup.php?codigo=5272787.

13. Colegio Mexicano de Ortopedia y Traumatología AC. Carta a la membresía sobre consultas a distancia. 2020. (Consultado el 25/10/2020.) Disponible en: http://www.smo.edu.mx/pdf/Opinion_Consulta_Online.pdf.

14. Eguia H, Vinciarelli F, Villoslada-Muñiz RL, Sanz-García FJ. Consulta no presencial en tiempos de coronavirus: información para médicos de Atención Primaria. Medicina de Familia. SEMERGEN. Digital 23/10/2020. (Consultado el 25/10/2020.) Disponible en: https://www.elsevier.es/es-revista-medicina-familia-semergen-40-avance-resumen-consulta-no-presencial-tiempos-coronavirus-S1138359320303129?newsletter=true\&coronavirus.

15. Declaración de la AMM sobre la ética de la telemedicina. Julio 2, 2020. (Consultado el 25/10/2020.) Disponible en: https://www.wma.net/es/policies-post/declaracion-de-la-amm-sobre-la-etica-de-la-telemedicina/.

16. Inspectorate $\mathrm{C}$. Information pack for video consulting in care home. Versión 2, 2019. (Consultado el 25/10/2020.) Disponible en: https://hub. careinspectorate.com/media/3633/care-home-resource-pack.pdf.

17. Boletín CONAMED- OPS. Abril 2016. El acto médico. Error y la mal praxis. (Consultado el 25/10/2020.) Disponible en: http://www.conamed. gob.mx/gobmx/boletin/pdf/boletin5/acto_medico.pdf.

18. Luna-Tomás MA, Margelí-Vila M, Ríos-Gozálvez C. Influencia de la pandemia por enfermedad por coronavirus 2019 en el manejo del cáncer de mama. Clin Invest Ginecol Obstet. 2020;47:89-90.

19. Moguel R, Samaniego V, Cabrera C, Chacek S, Estrada A. Missing heart attacks during confinement. Cardiovasc Metab Sci. 2020;31:38-9.

20. Los efectos de la pandemia del COVID-19: desafíos para la salud sexual y reproductiva. (Consultado el 25/10/2020.) Disponible en: https://www.gob.mx/conapo/articulos/participa-conapo-en-dialogo-virtual-sobre-los-efectos-de-la-pandemia-del-covid-19-desafios-para-la-salud-sexual-y-reproductiva?idiom=es.

21. Vavouraki E. The impact of COVID-19 pandemic on the healthcare of premature babies. Eur J Midwifery. 2020;4(May). doi:10.18332/ ejm/122385

22. Vallejo-Barón J. Embarazo en adolescentes, complicaciones. Rev Med Costa Rica y Centroamerica. 2013;70:65-9.

23. Moguel-Ancheita S, Orozco-Gomez LP. Analysis of risk factors for neurodevelopment and visual functions in the preterm infant to establish an early detection and treatment. Arch Ophthalmol Eye Disord. 2020;2:10-9. 